\title{
Soft mechatronics: an emerging design paradigm for the conception of intrinsically compliant electro-mechanical systems
}

\author{
Giovanni Berselli $\cdot$ Xiaobo Tan $\cdot$ Rocco Vertechy
}

Published online: 26 October 2015

(C) Springer Science+Business Media Dordrecht 2015

\section{Preface}

Soft mechatronics is an emerging field of science and technology, which focuses on the study of machines that synergistically combine highly compliant mechanical structures, electronics and control [1,2]. It pushes the limits of the consolidated mechatronic design practice [3], in an attempt to:

- Further increase flexibility and functionality of state-of-the-art electro-mechanical systems, while potentially simplifying design solutions [4]. For a number of important applications, conventional rigid machines are too heavy, energy-inefficient, expensive and relatively complex.

- Explore unconventional manufacturing methods, with the aim of improving parts integration and

G. Berselli ( $($ )

DIME - Department of Mechanics, Energetics,

Management and Transportation, Università di Genova,

Via allOpera Pia 15/A, 16145 Genoa, Italy

e-mail: giovanni.berselli@unige.it

\section{Tan}

Department of Electrical and Computer Engineering,

Michigan State University, 428 S. Shaw Lane, Rm. 2120

Engineering Building, East Lansing, MI 48824, USA

e-mail: xbtan@msu.edu

\section{R. Vertechy}

DIN - Department of Industrial Engineering, Università di Bologna, Viale Risorgimento 2, 40126 Bologna, Italy

e-mail: rocco.vertechy@unibo.it reducing assembly complexity [5, 6]. Most mechatronic devices are manufactured by combining "off-the-shelf" components (such as bearing, gears, and pulleys) and custom machined parts. However, being composed of a large number of parts, these complex devices might fail in a unpredictable way rather than gently degrade during their functioning.

- Design and produce devices with better safety properties when interacting with humans [7]. Many industrial systems are designed to be fast and stiff in order to achieve precise position control during highly dynamic motions. Such intrinsically stiff mechanical structures can pose safety hazards when interacting with unstructured environments or humans.

A major goal for the field of soft mechatronics is to develop soft, robust and affordable multi-functional components that physically interact with the surrounding, including humans, in a safe and effective manner. From an engineering perspective, soft mechatronics may be envisaged as an alternative design paradigm, in which flexible mechanisms, stretchable electronics, soft sensori-motors and power supplies are integrated to form intrinsically compliant electro-mechanical systems. These devices and systems are expected to open up new technological horizons and opportunities, with potential applications in healthcare, environment, security, renewable energy, and entertainment. Examples include wearable medical devices, biomimetic 
robots, environmental monitoring systems, and energy-harvesting machines.

In their present forms, soft mechatronic devices are typically compact systems composed of a dense assembly of elastic mechanical elements or mechanisms, electrical machines, and a variety of sensors. They are often made with metallic and magnetic materials. In their futuristic and most ambitious forms, soft mechatronic devices are solid-state systems featuring a large number of compliant transduction elements (namely, sensors, actuators, and generators) that are highly distributed in space and integrated in function. They are likely to be made with multifunctional polymers that can be activated via a number of different physico-chemical stimuli and feature deformation-dependent transduction properties.

As a natural evolution of the traditional mechtronic design paradigm, soft mechatronics requires the concurrent and synergic fusion of models, methods, and tools from several engineering disciplines. In this context, the purpose of this Special Issue is to provide a sample of recent research accomplishments in the field, with a particular focus on the multidisciplinary methodological frameworks shared by mechanical and electrical engineers when dealing with theoretical or technological issues in soft mechatronics. Overall, fifteen articles are included, specifically reporting on current research in soft transducers, mechatronic systems based on highly compliant mechanisms, and novel strategies for the effective control of highly deformable bodies. The aim is to project an insightful big picture of current trends and future challenges for the successful conception and subsequent development of the next-generation compliant electro-mechanical systems.

Acknowledgments The Guest Editors would like to thank all the researchers, from academia and industry, who have submitted their original research results to this Special Issue. We also would like thank the Editor-in-Chief, Prof. Luigi Gambarotta, and the Associate Editor, Prof. Eugenio Dragoni, for their support and help throughout the process. In parallel, we would like to acknowledge the support from the ASME Dynamic Systems and Control Division, Technical Committee on Mechatronics, along with the ASME Aerospace Division, Technical Committee on Modeling, Simulation, and Control of Adaptive Systems, for the valuable help in disseminating the call-for-papers. Finally, we would like to thank Kokila Kothandaraman, from the Springer Editorial Office, and the numerous referees for their expertise and hard work dedicated to the reviewing task.

\section{References}

1. Majidi C (2013) Soft robotics: a perspective-current trends and prospects for the future. Soft Robot 1(1):5-11

2. Kim S, Laschi C, Trimmer B (2013) Soft robotics: a bioinspired evolution in robotics. Trends Biotechnol 31(5): 287-294

3. Isermann R (2003) Mechatronic systems. Springer, London

4. Popovich D (1998) Mechatronics in engineering design and product development. CRC Press, Boca Raton

5. Gibson I, Rosen D, Stucker B (2014) Additive manufacturing technologies: 3D printing, rapid prototyping, and direct digital manufacturing. Springer, Berlin

6. Krar S, Gill A (2003) Exploring advanced manufacturing technologies. Industrial Press Inc., South Norwalk

7. Bicchi A, Tonietti G (2004) Fast and "soft-arm" tactics [robot arm design]. IEEE Robot Autom Mag 11(2):22-33 